\title{
Pratiques
}

Linguistique, littérature, didactique

183-184| 2019

oralité, littératie

\section{À l'école maternelle, une entrée différentiée dans l'écrit entre oralité et littératie}

Ariane Richard-Bossez

\section{(2) OpenEdition}

Journals

Édition électronique

URL : http://journals.openedition.org/pratiques/7533

DOI : 10.4000/pratiques.7533

ISSN : 2425-2042

Éditeur

Centre de recherche sur les médiations (CREM)

Référence électronique

Ariane Richard-Bossez, « À l'école maternelle, une entrée différentiée dans l'écrit entre oralité et littératie », Pratiques [En ligne], 183-184 | 2019, mis en ligne le 30 décembre 2019, consulté le 11 octobre 2020. URL : http://journals.openedition.org/pratiques/7533 ; DOI : https://doi.org/10.4000/ pratiques. 7533

Ce document a été généré automatiquement le 11 octobre 2020

(c) Tous droits réservés 


\title{
À l'école maternelle, une entrée différentiée dans l'écrit entre oralité et littératie
}

\author{
Ariane Richard-Bossez
}

\section{Introduction}

1 Oralité et littératie se réfèrent à des opérations intellectuelles rendues possibles par les ressources spécifiques à l'écrit et à l'écriture pour la littératie ou relatives à l'oral pour l'oralité. Ainsi on peut considérer la littératie comme relevant à la fois d'une «raison graphique » (Goody, 1979 [1977]), d'une organisation matérielle et sociale raisonnée fondée sur les propriétés spatiales « chronotopiques » de l'écrit (Laparra \& Margolinas, 2016) et, de manière plus étendue, d'un rapport symbolique et second à la langue, au monde et à ses objets (Bautier, 2009). De ce fait elle s'inscrit dans ce que J.-M. Ferry (2004) nomme la " grammaire propositionnelle ", c'est-à-dire un mode d'appréhension du monde ayant pour racine sur une approche rationnelle et objective. L'oralité, quant à elle, s'appuie sur des opérations cognitives reposant sur la « coprésence des corps et des objets » (Laparra \& Margolinas, 2016), fortement "routinisées» (Laparra \& Margolinas, 2012) et contextualisées. Elle repose également sur des « grammaires infraverbales » (Ferry, 2004) caractérisées par leur dimension non conceptuelle, s'appuyant sur des informations procurées sur un mode perceptif et impressionnel et où la communication et la compréhension se fondent sur l'imitation et l'indication.

2 L'apprentissage de l'écrit à l'école maternelle, tel qu'il est présenté dans les programmes scolaires, s'inscrit dans une logique littératiée. Il y est ainsi précisé que :

Il appartient à l'école maternelle de donner à tous une culture commune de l'écrit. Les enfants y sont amenés à comprendre de mieux en mieux des écrits à leur portée, à découvrir la nature et la fonction langagière de ces tracés réalisés par quelqu'un pour quelqu'un, à commencer à participer à la production de textes écrits dont ils explorent les particularités. En fin de cycle, les enfants peuvent montrer tous ces 
acquis dans leurs premières écritures autonomes. (Ministère de l'Éducation nationale et de la Jeunesse, 2015, p. 7)

Dans cet article, nous proposons de répondre à l'interrogation suivante : comment, au cours des activités relatives à l'écrit en maternelle, circulent des opérations relevant à la fois de l'oralité et de la littératie et comment s'articulent-elles, ou non, dans les apprentissages des élèves? Pour ce faire, on partira des résultats d'une recherche en sociologie de la pédagogie (Bernstein, 2007 [1996]), croisant sociologie de l'éducation et sociologie de la connaissance, portant sur la construction des savoirs et ses inégalités à l'école maternelle (Richard-Bossez, 2015). Le propos sera illustré à partir de données issues d'une centaine d'heures d'observations réalisées lors d'une enquête de terrain de type ethnographique entre décembre 2010 et juin 2011 dans six classes de grande section situées dans des contextes sociaux variés (trois classes en "éducation prioritaire ", trois classes « ordinaires ») de la région toulonnaise. Trois dimensions du processus pédagogique seront développées. On commencera par exposer la manière dont les savoirs relatifs à l'écrit sont présentés aux élèves. Il s'agira ensuite de montrer comment les élèves se saisissent cognitivement de ces savoirs et les opérations intellectuelles qu'ils mobilisent pour le faire. Enfin, on s'intéressera aux échanges qui se nouent entre enseignants et élèves, notamment pour les élèves qui n'arrivent pas à saisir les savoirs en jeu sur le registre littératié attendu par l'école. De manière transversale, on interrogera les différenciations qui sont perceptibles dans l'apprentissage de l'écrit.

\section{La présentation des savoirs de l'écrit aux élèves : une dimension littératiée peu explicitée}

4 À l'école maternelle, les savoirs sont essentiellement médiatisés par la présentation orale $^{1}$ que l'enseignant ${ }^{2}$ en fait aux élèves lors de moments de regroupement où sont exposées les activités que les élèves réaliseront ensuite en petits groupes dans les ateliers. En analysant ces formes de présentation orale, il est possible de dégager trois entrées mobilisées par les enseignants qui s'appuient de manière variable sur les ressources de la littératie et de l'oralité. Les enseignants observés ont recours à sur l'ensemble de ces modes, même si des tendances plus fortes peuvent s'exprimer chez les uns ou les autres.

\section{Une présentation centrée sur les savoirs}

5 Une première entrée mobilisée par les enseignants pour présenter les activités d'apprentissage donne une place centrale aux savoirs. Ceux-ci sont alors exposés de manière relativement abstraite, en insistant sur leur caractère universel et dépassant la seule situation en jeu. L'attention est alors particulièrement portée sur les critères permettant de reconnaître ce savoir. L'attitude et le comportement attendu de la part des élèves ainsi que les procédures à mettre en œuvre pour réaliser l'activité demandée sont également fortement explicitées. L'accent y est mis davantage sur la dimension métalangagière de l'écrit que sa dimension plus sociale. On retrouve donc dans ce mode de présentation une logique fortement littératiée. Cette première entrée est cependant peu fréquente dans nos observations (environ $15 \%$ des cas), on la retrouve davantage lors d'activités de type dirigé où l'enseignant est présent en continu auprès des élèves. 
6 On peut illustrer ce mode de présentation par un atelier où, dans le cadre d'un travail plus global autour de la lecture d'un conte, l'enseignante propose aux élèves d'écrire une phrase à partir d'étiquettes-mots. Elle leur explique l'activité comme suit :

Une phrase, c'est quelque chose... c'est des mots... des mots les uns après les autres qui veulent dire quelque chose, qui ont un sens. [...] Une phrase, ça se termine toujours par un... point. [...] quand on écrit, on commence toujours la phrase par une majuscule. Bon, ça vous le mettez dans un coin de votre petite tête, vous le gardez bien précieusement, et puis ça vous servira plus tard.

7 La dimension littératiée est ici mise en avant par l'utilisation de termes relatifs à l'écrit («phrase », « mot », « point », « majuscule »), par la référence au sens graphique (« les uns après les autres ", « ça se termine », « on commence ») ainsi que par l'insistance sur le caractère générique et permanent de ces caractéristiques («une phrase», « toujours », « ça vous servira plus tard »).

\section{Une présentation centrée sur l'activité}

8 Une deuxième entrée mobilisée pour présenter les activités d'apprentissage de l'écrit consiste à mettre l'accent sur l'activité globale proposée, les savoirs eux-mêmes devenant alors plus secondaires. Par conséquent, dans ces cas-là, le savoir est davantage un élément relatif à une activité plus large sans en être l'objet central. Il devient également plus localisé dans la mesure où les explicitations données restent relatives au cas proposé sans insister sur ce qui pourrait être transférable à d'autres situations. Dans cette présentation, c'est le sens global de la situation qui est privilégié plus que les connaissances requises. La dimension épistémique des savoirs est ainsi davantage invisibilisée alors que la dimension sociale est plus présente mais de manière très localisée au groupe classe. Ce mode de présentation s'appuie donc de manière plus forte sur les ressources de l'oralité, même si des dimensions littératiées peuvent également être présentes. Cette entrée est la plus représentée dans nos observations (environ la moitié des cas), elle est particulièrement mobilisée quand l'enseignant travaille autour d'un thème ou d'un projet ou lors de la présentation d'activités censées être déjà connues des élèves.

9 Ainsi, dans le cadre d'un travail autour du Japon, une enseignante propose une activité de transcription de mots en minuscules d'imprimerie en lettres capitales. Elle présente cette activité aux élèves à partir de mots issus d'un album lu en classe :

Je vous ai mis les mots de l'album de Yumi [personnage principal de l'album] et je vais vous demander de les écrire en majuscules au crayon à papier. [...] Alors, vous regardez ... Alors, ici, il y a écrit Yumi, je vais vous demander dessous, d'écrire Yumi en majuscules. Là, je l'ai écrit en script. [...] L'alphabet vous l'avez en bas [de la fiche d'exercices], en détaché et en majuscules. Donc, si par exemple le Y, vous ne savez pas comment on le fait en majuscule, vous cherchez le $Y$ en bas, et dessous il est en majuscule. Donc, vous regardez en bas [de la fiche] si vous savez pas faire la lettre, d'accord?

10 Dans cette présentation, l'objet principal semble être les mots de l'album à transcrire («Yumi ») plus que la correspondance entre les deux formes d'écriture de l'alphabet qui sont évoquées ("majuscules», «script») mais de manière imprécise et peu explicitée. On retrouve aussi des logiques relatives à l'oralité puisque la situation s'appuie sur des expériences partagées par le groupe-classe et des explications qui font une place importante aux références déictiques (« ici », « dessous », « là », « en bas »...). La dimension littératiée est également présente mais uniquement dans son aspect 
graphique, par la présence sur la fiche d'un tableau de correspondance entre alphabet en minuscule d'imprimerie et alphabet en lettres capitales. En revanche, la dimension plus abstraite (permanence des lettres au-delà des formes d'alphabets utilisées, raison d'être de ces différents alphabets...) reste implicite.

\section{Une présentation centrée sur les tâches}

11 Enfin, la troisième forme de présentation discernable est une entrée axée sur les tâches. Les savoirs tendent ici à disparaître en tant que tels et l'ancrage dans les circonstances de la situation devient l'élément principal. L'objectif n'est plus, comme dans le cas précédent, de réaliser une activité globale mais d'effectuer des tâches ponctuelles fortement contextualisées. L'apprentissage visé tend alors à s'effacer au profit d'une action qui devient son propre objectif. Ainsi, dans ce mode de présentation, ni la dimension épistémique, ni la dimension sociale de l'écrit ne semblent présentes, seul l'exercice à réaliser apparaît être retenu. Ce sont donc essentiellement des ressources liées à l'oralité qui sont mobilisées. Ce mode de présentation est assez fortement présent dans notre corpus puisqu'il représente environ un tiers des cas observés.

C'est le cas dans cette activité où l'enseignante propose de classer des mots en fonction de leur initiale en attachant une importance plus forte aux tâches à réaliser (" découper », « coupe sur le trait ») qu'aux savoirs en jeu (le terme " initiale » n'est pas énoncé et la part cognitive de la tâche est effectuée par l'enseignante qui donne les réponses aux élèves) :

Alors les bleus, je vous montre : il y a un travail avec des mots, des étiquettes-mots qu'il faut découper, toutes ces étiquettes, là, on coupe sur le trait, là, tout droit. On coupe sur le trait et ensuite il y a quatre bulles, mais il y a des petites étiquettes, mais il n'y a rien marqué sur les étiquettes, alors il faut bien regarder la première lettre de chaque mot, il y a des mots qui vont commencer par la lettre P, des mots par la lettre... des mots par la lettre... (elle dit les noms des lettres avec les élèves) $F$. Et des mots par la lettre $C$. Donc et après je vois que là aussi ça commence par $R$, par P, par R, par C, R, F, C, F [...] Alors, écoutez-moi, dans une bulle, le pétunia oui c'est une fleur. Dans une des bulles, je vais mettre tous les mots qui commencent par la lettre $\mathrm{P}$, celui-là, celui-là, celui-là, je cherche, il doit y en avoir 1, 2, 3 (montre les mots concernés). Je les mets là [...] donc écoutez bien, j'explique. Les trois qui commencent par le $\mathrm{P}$, je les mets dans la même bulle, et ici j'écris $\mathrm{P}$, les trois qui commencent par le $\mathrm{C}$, je les mets tous les trois ensembles : carotte, chou-fleur et puis chou et courgette là, dans la petite étiquette, je mets la lettre $C$. Les trois qui commencent par le $\mathrm{R}$ : radis, riz et raisin, je vais les mettre ici et je vais mettre la lettre R. Et les derniers, c'est ceux qui commencent par $\mathrm{F}$ : fraise, fève...

Ici, même si les noms des lettres sont cités, c'est essentiellement sur des ressources liées à l'oralité, spécifiques au contexte de l'activité (les mots montrés par l'enseignante), que la présentation se fonde.

La manière dont les savoirs relatifs à l'écrit sont présentés aux élèves laisse donc une place relativement peu importante aux opérations littératiées au profit de celles relevant de l'oralité. On peut mettre cela en lien avec le fait que les enseignants oscillent dans leurs présentations entre la formulation des notions abstraites en jeu, relevant de la littératie, et les situations concrètes dans lesquelles ces notions sont 
mobilisées, fondées sur l'oralité. Cela a pour conséquence de laisser cette dimension littératiée le plus souvent dans l'implicite et, de ce fait, de risquer de la rendre imperceptible pour les élèves qui ne savent pas la repérer.

\section{L'appropriation des savoirs de l'écrit par les élèves : un appui sur des ressources différenciées}

15 Il s'agit maintenant de s'intéresser aux opérations cognitives que les élèves mobilisent dans les activités d'apprentissage de l'écrit, c'est-à-dire à la manière dont les savoirs sont saisis intellectuellement et aux connaissances que les élèves utilisent pour le faire. Trois registres faisant une place variable aux opérations littératiées peuvent être distingués. On pourrait avoir tendance à penser que les registres d'interprétation mobilisés par les élèves varient en fonction de la manière dont l'activité leur a été présentée. Or, on observe que chaque atelier donne lieu à un éventail d'interprétations couvrant généralement l'ensemble des registres présentés, et ceci de manière relativement indépendante du mode de présentation des savoirs par l'enseignant, comme nous le verrons à partir d'exemples tirés des trois situations présentées dans la première partie de l'article.

\section{Une appropriation de l'écrit sur un registre littératié}

16 Un premier registre d'interprétation mobilisé par certains élèves est un registre littératié. Celui-ci recouvre des dimensions multiples, correspondant aussi bien aux spécificités techniques de la langue française, qu'à sa dimension graphique ou au rapport second au monde qu'elle implique. Ce registre correspond donc aux attentes des programmes scolaires. Il s'exprime par la maittrise seconde des unités langagières que sont les sons, les lettres, les mots ou encore les phrases. Les bases de la lecture et des comportements de lecteurs chevronnés associant lettres et sons ou saisissant la dimension littéraire de l'écrit y sont également manifestes. Les élèves considérés comme les meilleurs ont recours à ce registre de façon quasi systématique, mais cela correspond à une minorité d'élèves dans les classes. On peut noter, que cette dimension littératiée de l'écrit se construit également dans la sphère familiale, comme en témoignent certains comportements: une élève apportant à l'école un manuel d'apprentissage de la lecture acheté dans le commerce, un autre expliquant que sa mère lui a acheté des albums d'un auteur de littérature jeunesse lu en classe, par exemple.

17 Ce mode d'appropriation des savoirs de l'écrit se retrouve dans les trois situations présentées dans la première partie de cet article, bien que la dimension littératiée y ait été différemment explicitée. Ainsi, dans l'atelier relatif à la composition de phrases présenté en se centrant sur les savoirs en jeu, Rémi, qui est considéré comme un très bon élève, propose à l'oral une phrase respectant les codes linguistiques de l'écrit: "L'esclave tombe dans le fleuve». Il reconstitue sans erreur sa phrase à l'aide des étiquettes-mots que lui a donné l'enseignante et l'illustre par un dessin correspondant au sens exprimé par la phrase. Au cours de l'atelier de transcription de mots en minuscules d'imprimerie en lettres capitales et présenté en se centrant sur l'activité par l'enseignante, Chérif, dès le début de l'activité, complète sa fiche seul et sans faire d'erreurs, qui plus est sans se référer aux alphabets en bas de la page. Les 
commentaires qu'il fait au cours de son travail montrent qu'il connaît déjà les lettres dans différentes écritures et certaines correspondances entre les lettres et les sons : il essaie de lire le mot «Bamboo » en prononçant « bbb » ou précise à son voisin que dans le mot « éventail », la lettre $\mathrm{N}$ ne « s'entend pas », que « c'est un N muet [en fait associé au A pour faire le son "an"] ", mais qu'il faut l'écrire « quand même ». Dans l'exercice de classement de mots en fonction de leur initiale qui a été présenté en se centrant sur la tâche en jeu, Kali déchiffre le mot «riz» en prononçant le son de la lettre $Z$ et demande « rizzz, ça veut dire quoi rizzz ? ». L'enseignante lui ayant répondu « c'est le riz », il note comme pour lui-même " $A$ h, un $Z$ ».

Le fait que ce mode d'interprétation soit faiblement représenté dans les classes signifie à la fois que les élèves de maternelle en sont potentiellement capables, et que les situations scolaires ne permettent pas à tous les élèves de l'exprimer.

\section{Une appropriation des savoirs de l'écrit sur un registre familier}

Un deuxième registre d'interprétation des savoirs mis en œuvre dans les activités de la classe relève du familier. Les savoirs sont alors appréhendés comme des éléments du quotidien, se fondant sur des usages courants semblables à ceux relatifs aux autres sphères de la vie de l'enfant, sans que leur soit attribué un statut plus scolaire. On est ici dans le registre de l'oralité. Au regard des apprentissages visés par les programmes scolaires, ce registre familier apparaît donc en décalage. En général, dans ce genre de situations, l'interprétation des savoirs se fonde sur le rattachement entre un élément de la situation scolaire et un élément lui étant extérieur, suivant une logique de type associative (Ferry, 2004). Ces liens peuvent être de différents ordres : relatifs à une expérience personnelle de l'enfant, une association de sens, un élément de l'environnement familier, ou la pratique langagière courante. Ce registre familier se distingue ainsi du registre littératié caractérisant les attentes des programmes officiels. De la sorte, sa mobilisation par les élèves traduit une non-perception des attentes propres au milieu scolaire.

On retrouve ce mode d'appréhension des savoirs de l'écrit dans les situations que nous avons présentées plus haut. Par exemple, dans l'atelier relatif à l'élaboration de phrases, Kenza formule une phrase ayant du sens mais qui s'appuie sur des tournures orales, doublant les substantifs d'un article redondant à l'écrit : « Le chasseur il dit que l'esclave elle est tombée dans le fleuve ", ce qui montre qu'elle ne maîtrise pas et ne perçoit pas les propriétés attendues d'une phrase écrite. Dans la deuxième situation fondée sur la transcription de mots en majuscules d'imprimerie, Asma, remplit sa fiche avec beaucoup d'application, son regard se déplace des cases à compléter aux alphabets-référents dans des allers-retours continuels, elle transcrit les lettres une à une, témoignant $\mathrm{du}$ fait qu'elle mobilise des opérations associatives qui restent fortement reliées au tableau de correspondance entre alphabets présent sur sa fiche. Dans la troisième situation, une partie de la discussion qui a eu lieu au cours de la présentation de l'atelier de classement de mots en fonction de leur initiale plusieurs élèves ont fait des commentaires relatifs au mot "fève", prenant en compte leur expérience personnelle : "c'est comme ce qui est dans les galettes », « dans mon jardin, moi j'en ai aussi », "on les ouvre et y a des ronds verts »... montrant ainsi une appréhension, au moins en partie, fondée sur l'aspect familier de l'activité proposée. 
21 Ce registre familier est fortement présent dans les classes. Cela souligne notamment une des particularités de l'apprentissage de la langue et de la non-évidence pour les élèves du passage entre les usages de la langue dans la vie courante et ses usages plus métacognitifs attendus à l'école.

\section{Une appropriation des savoirs de l'écrit sur un registre pragmatique}

Enfin, un troisième mode d'interprétation des savoirs observable dans les situations scolaires est fondé sur un registre pragmatique. Ce registre est également relativement fréquent dans nos observations et est souvent celui auquel ont recours les élèves les plus en difficulté par rapport aux apprentissages scolaires. Ce mode d'interprétation s'observe, d'une part, par un appui sur des opérations perceptives infra-verbales (Ferry, 2004), et d'autre part, par la priorité donnée à l'action et à son résultat. Ce troisième registre d'interprétation des savoirs s'appuie de la sorte sur les dimensions les plus pragmatiques des activités. Les opérations perceptives, et notamment celles fondées sur l'aspect visuel des supports proposés aux élèves, s'y révèlent particulièrement importantes. Les actions nécessaires à la réalisation de la tâche demandée ainsi que la forme du résultat final recherché sont également des éléments qui caractérisent fortement ce registre. Le fait que les élèves considérés comme les plus en difficulté s'appuient sur ce registre pragmatique semble indiquer qu'on y a recours quand on ne peut pas faire appel à d'autres ressources. L'accent qui est mis sur les actions à réaliser et la conformité du résultat à ce qui est attendu traduisent également la volonté de s'ajuster aux attentes de l'activité et, par conséquent, une forme de recherche des critères de réussite.

C'est le cas de Nolan et Samir qui ne parviennent pas à énoncer une phrase comme demandé par l'enseignante et répètent certains noms de personnages entendus lors de la présentation de l'atelier ("Kora », "Emmée », " Nourou ») mais sans les intégrer dans une phrase. La présentation de l'atelier fortement littératiée n'a donc pas été suffisante pour que ces élèves se saisissent de cette dimension. Dans le deuxième atelier, Adam complète sa fiche en recopiant les lettres en minuscules, sans les transcrire en capitales et en s'appliquant à reproduire les détails des empattements des lettres liés à la police de caractère utilisée sur la fiche. Enfin, dans le troisième atelier, Mia, découpe les étiquettes, les pose à côté de sa fiche et les colle dans l'ordre où elles sont posées, 3 par 3 sans prendre en compte les initiales des mots, s'appuyant sur l'aspect pragmatique de la tâche.

Ces éléments témoignent de la variété des ressources que les élèves mobilisent dans les activités scolaires pour saisir les savoirs de l'écrit. Cela montre, d'une part, la variabilité des expériences antérieures des élèves, que celles-ci soient scolaires ou familiales; et d'autre part, les écarts existants entre les élèves par rapport aux opérations littératiées définies dans les programmes scolaires. Au travers de ces exemples, on voit également que la manière qu'ont les élèves de s'approprier les savoirs de l'écrit se fait de façon relativement indépendante de la manière dont l'exercice a été présenté par l'enseignant. Pour certains élèves, même si l'exercice a été présenté sans faire de référence explicite aux savoirs en jeu, ils sauront les saisir sur un registre littératié. 
Symétriquement, même si la dimension littératiée a été davantage mise en avant, ce n'est pas pour autant que les élèves - en particulier ceux en difficulté - sauront s'en saisir. Cela amène à penser que la manière dont les savoirs sont investis est plus liée au niveau d'intégration de ces savoirs par les élèves qu'à la manière dont ils sont présentés.

\section{Des interactions entre enseignant et élèves qui peuvent participer à la révision des savoirs}

Cependant, si les registres d'appropriation des activités d'apprentissage de l'écrit semblent varier plutôt en fonction des connaissances déjà maitrisées par les élèves qu'en fonction de la manière dont ces savoirs sont présentés par l'enseignant, d'autres dimensions du processus pédagogique sont susceptibles de faire évoluer les conceptions initiales des élèves. C'est notamment le cas des interactions qui se nouent entre enseignants et élèves au cours des activités d'apprentissage ${ }^{3}$. Soulignons que, pour que des interactions aient lieu, certaines conditions doivent être réunies sur le plan de l'organisation pédagogique de la classe, par exemple le fait que l'enseignant puisse passer dans les ateliers ou que les élèves puissent se déplacer. Cela n'est pas toujours le cas, mais quand les conditions sont réunies pour que des interactions entre enseignants et élèves aient lieu, selon les modalités que prennent ces interactions, leurs effets sur les apprentissages peuvent varier en termes de possibilités de révision (Marquis, 2001) ${ }^{4}$ des savoirs des élèves et ce particulièrement pour les élèves dont l'interprétation première des savoirs ne correspond pas aux attendus littératiés visés.

\section{Des interactions qui maintiennent les apprentissages dans des registres non littératiés}

Certaines interactions ne semblent pas amener d'élément déclencheur permettant la reprise des savoirs initiaux des élèves pour aller vers un registre plus littératié.

C'est le cas, d'une part, quand les interactions ne se centrent pas sur les difficultés cognitives de l'élève. Par exemple pour Kader qui a des difficultés à retrouver les mots écrits en différentes écritures sur sa fiche : il regarde les fiches de ses voisins, ne sait manifestement pas quoi faire, joue avec ses crayons de couleur. La maîtresse vient et lui demande de lui montrer les mots correspondants. Les réponses de Kader sont incorrectes. L'enseignante quitte l'atelier en maugréant. Kader regarde alors sa feuille en se tordant les mains. Cela s'observe aussi quand les échanges se centrent sur l'incitation à la participation des élèves : «Allez Samir !", « continue », "Zinedine, tu cherches un peu ? »... Ou quand les erreurs de l'élève sont pointées mais sans chercher à les reprendre sur un plan cognitif. Par exemple, comme quand l'enseignant enlève des étiquettes-mots mal positionnées ou gomme des erreurs sans donner d'explications supplémentaires.

C'est le cas, d'autre part, quand les interactions sont fondées sur des registres non littératiés. Les reprises proposées par l'enseignant peuvent se centrer sur l'aspect pragmatique de la tâche, en donnant des précisions méthodologiques ou organisationnelles (organiser ses étiquettes sur la table par exemple) ou en découpant la tâche en phases fractionnées. Mais cela peut également prendre la forme d'une 
réduction cognitive de la tâche pour l'élève, en réduisant le nombre d'éléments à traiter. Ainsi quand Kader doit reconstituer des phrases à partir d'étiquettes-mots, l'enseignante lui dit « ça suffit de découper je crois, regarde, tu as déjà tout ça à placer, c'est déjà beaucoup. Ça on va mettre de côté pour l'instant (elle enlève le reste des étiquettes) ». Soit en prenant en charge la part cognitive de la tâche par l'enseignant. Par exemple, quand Nolan ne parvient pas à formuler une phrase, c'est l'enseignante qui la formule : "Qu'est-ce que tu préfères écrire ? "Nourou est amoureux" ou "Emmée est amoureuse" ?». Ou bien dans l'exemple suivant ou Roger interroge l'enseignante qui lui donne les réponses directement:

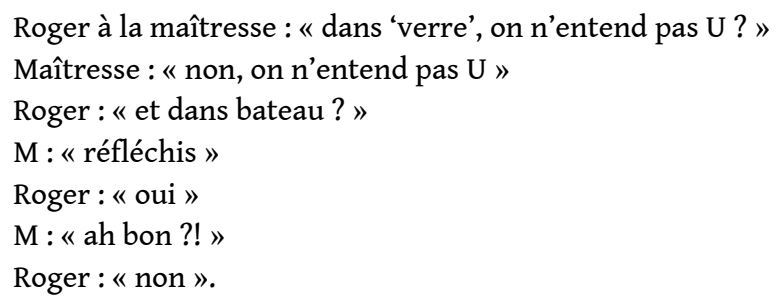

Cela peut également se faire en modifiant la nature des opérations cognitives demandées à l'élève. Ainsi, lorsque l'enseignante cherche à aider Nolan qui n'arrive pas à trouver le mot "vendredi » parmi les étiquettes des jours de la semaine, elle commence par dire "Alors, vvvendredi, quel est le jour qui commence par le vvv de vendredi ? » en lui donnant un indice littératié fondé sur le son produit par l'initiale du mot. Cet indice ne semblant pas permettre à Nolan de donner la bonne réponse, elle continue en s'appuyant sur le nom de la lettre "Nolan, où est-ce qu'il y a le V? ». N'obtenant toujours pas la réponse souhaitée, elle finit par lui dire «Je te montre la forme $\mathrm{du} \mathrm{V}$ » en faisant la forme de la lettre avec ses mains.

Ces modes de reprise peuvent parfois amener l'élève à fournir l'apparence du résultat escompté, mais au regard des opérations cognitives visées, ils ne permettent pas aux élèves de réviser leurs modes d'appréhension des savoirs vers un registre plus littératié.

\section{Des interactions qui proposent des ouvertures vers l'apprentissage de la dimension littératiée}

On peut aussi déceler des reprises fondées sur des registres plus réflexifs qui semblent ouvrir des possibilités d'accès à des registres d'appréhension des savoirs plus littératiés.

C'est le cas quand l'enseignant signale, explicite et argumente à l'élève la non validité de sa réponse en vue de lui faire prendre conscience de la transformation à effectuer. C'est également le cas quand l'échange se centre sur la réflexivité de l'élève en l'accompagnant dans la transformation de ses réponses, mais (à la différence du cas précédent) en lui laissant une part de la tâche cognitive à effectuer lui-même. Cela peut prendre la forme de processus d'étayage-désétayage visant à transférer progressivement à l'élève la part cognitive du travail à effectuer, ou bien s'organiser autour de questions ouvertes qui visent à guider l'élève et à l'amener à réfléchir par luimême à la réponse à fournir. Enfin, cela se retrouve aussi dans les interactions construites sur l'explicitation des critères de savoir à partir d'arguments cognitifs justifiant la nécessité de reprendre l'interprétation première des savoirs de l'élève. Cela s'observe par exemple quand l'enseignante donne des indices se fondant sur des 
critères cognitifs pour aider Samir - un élève fréquemment en difficulté - à retrouver dans ses étiquettes le mot « Kora »:

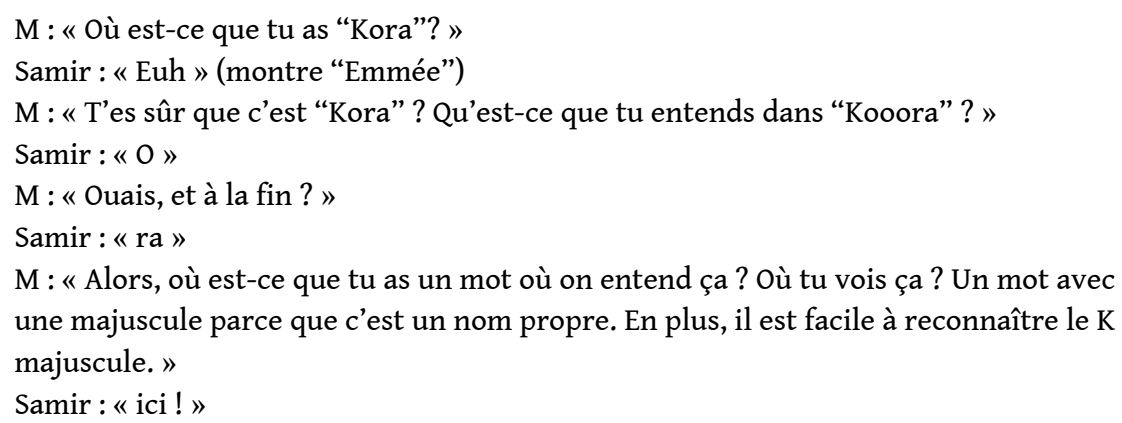

L'analyse de ces situations montrent que, pour des apprentissages variés, cela permet à des élèves, y compris ceux considérés comme les plus en difficulté, de reconsidérer leur interprétation initiale des savoirs en jeu pour aller vers une appropriation plus littératiée.

Cela peut également avoir lieu sous la forme d'échanges collectifs. D'une part par le recours de l'enseignant à des pairs, soit pour apporter une connaissance que certains maitrisent déjà, soit pour faire expliciter les connaissances en jeu dans la situation. D'autre part, cela peut se faire sous forme d'une élaboration commune de savoirs à partir des apports des différents membres du groupe, comme dans cette activité où les élèves déchiffrent une lettre reçue d'une autre classe :

$\mathrm{M}$ « Qui est-ce qui le voit le mot "bravo"? Qui est-ce qui a une idée où il est le mot bravo ? Thomas? Allez, vas-y, montre-nous (Thomas montre "travaillé"). Ha?»

Des élèves : « non »

$\mathrm{M}:$ «A votre avis, les autres?»

Les élèves : «non »

$\mathrm{M}:$ : Pourquoi ça peut pas être "bravo"?»

Un élève : "parce que y a pas un $B$ au début »

$\mathrm{M}:$ «Parce que y a pas un $\mathrm{B}$ au début»

Un élève : « $\mathrm{y}$ a pas un $\mathrm{R}$ »

$\mathrm{M}:$ : Y a pas le V non plus qu'on entend dans "bravvvo". Keanu, vas lui montrer ce que c'est. Est-ce que tu le vois "Brravvo". (Keanu hésite) Ah, c'est dur hein. » Keanu ne trouve pas, Anaïs vient l'aider.

$\mathrm{M}$ : «Eh ben oui, c'est ici. Fais voir Anaïs. (à Keanu) Qu'est-ce que tu en penses, qu'est-ce que tu penses, tu pensais à quoi toi ? (Keanu montre "bonjour") Alors non parce que c'est "bonjour", mais tu as raison, y a le B. »

Keanu s'approche, regarde la feuille intensément, semble ne pas savoir puis montre le bon mot (fier avec un sourire).

$\mathrm{M}:$ « Keanu l'a trouvé, très bien. Bravo, en plus je l'ai écrit en gros, hein "bravo” »

$\mathrm{M}:$ : Qu'est-ce qu'on entend à la fin de bravo?»

Des élèves : « le $0 . »$

$M:$ « Le $O$ et on le voit, alors je vais l'entourer là. »

Ce qui semble commun à ces situations où s'ouvrent des possibilités d'apprentissages plus littératiés, c'est l'appui sur ce que Ferry (2004) appelle la "grammaire délibérative ", c'est-à-dire des opérations critiques fondées sur l'explication, l'argumentation et la délibération. Cette grammaire se construit sur les ressources propositionnelles et littératiées mais va au-delà en permettant des formes d'autoréflexivité ainsi qu'un agencement et une reconnaissance de dispositions appartenant à d'autres logiques que la sienne ouvrant ainsi sur des possibilités d'« espace cognitif collectif » (Pandraud, 2012). 
Les interactions qui se nouent entre enseignants et élèves sont donc, davantage que les modes de présentation des savoirs, le lieu où s'ouvrent ou se ferment des possibilités de reprise des savoirs initiaux et pouvant amener à des processus de révision pour aller vers des modes d'appropriation de l'écrit plus littératiés. On observe cependant que ces moments de révision restent relativement aléatoires et ponctuels dans les classes, ne permettant pas de modifier réellement en profondeur les modes d'appréhension de l'écrit par les élèves.

\section{Conclusion}

37 Au fil de cet article, nous avons souhaité montrer comment s'élaborent les savoirs relatifs à l'écrit chez des élèves de maternelle, notamment au regard de la dimension littératiée attendue. Nous avons insisté sur le fait que ces apprentissages se construisent par la mise en relation de différentes dimensions : la présentation orale des savoirs par les enseignants, la manière dont ils sont appropriés par les élèves et les échanges qui peuvent se nouer entre enseignants et élèves au cours des activités. Nous avons également souligné qu'au cours de ces différents moments les appuis sur des opérations relevant de l'oralité ou de la littératie se mêlent de manière variée, mais souvent en insistant plus sur les premières que les secondes. Si l'on interroge la nature des apprentissages réalisés par les élèves, on observe des apprentissages différentiés. D'une part, pour les élèves possédant déjà des ressources littératiées, les activités proposées dans les classes sont l'occasion d'entraîner encore cette dimension. D'autre part, pour les élèves qui ne la maîtrisent pas, les situations proposées dans les classes permettent peu de la travailler, maintenant ainsi certains élèves à l'écart des opérations. L'entrée dans l'écrit à l'école maternelle apparait donc être un processus différencié (et différenciateur) où oralité et littératie ne s'articulent que de manière relativement ponctuelle.

\section{BIBLIOGRAPHIE}

BAUTIER, E. (2009). « Quand le discours pédagogique entrave la construction des usages littératiés du langage ». Pratiques 143-144, p. 11-26.

BERNSTEIN, B. (2007) [1996]. Pédagogie, contrôle symbolique et identité. Théorie, recherche, critique. trad. de l'anglais par G. Ramognino Le Déroff \& P. Vitale. Laval : Presses de l'université de Laval.

FERRY, J.-M. (2004). Les grammaires de l'intelligence. Paris : Cerf.

GOODY, J. (1979) [1977]. La Raison graphique. La domestication de la pensée sauvage. Trad. de l'anglais par J. Bazin et A. Bensa. Paris : Éditions de Minuit.

LAPARRA, M., \& MARGOLINAS, C. (2012). « Oralité, littératie et production des inégalités, scolaires ». Le Français Aujourd'hui 177, p. 55-64.

Pratiques, $183-184 \mid 2019$ 
LAPARRA, M. \& MARGOLINAS, C. (2016). Les premiers apprentissages scolaires à la loupe. Louvain-laNeuve : De Boeck.

MARQUIS, P. (2001). Révision, cognition et interaction. Paris : L'Harmattan.

Ministère de l'Éducation nationale et de la Jeunesse (2015). « Programme de l'école maternelle ». Bulletin officiel spécial $n^{\circ} 2$ du 26 mars 2015. En ligne : https://www.education.gouv.fr/au-bospecial-du-26-mars-2015-programme-d-enseignement-de-l-ecole-maternelle-3413.

PANDRAUD, N. (2012). L'exposition différenciée aux apprentissages du français en classe de sixième. Processus sociaux et épistémiques de la révision des savoirs. Une enquête par observation dans deux collèges marseillais. Thèse de doctorat en sociologie. Marseille : Université d'Aix-Marseille.

RICHARD-BOSSEZ, A. (2015). La construction sociale et cognitive des savoirs à l'école maternelle : entre processus différenciateurs et moments de démocratisation. Le cas des activités relatives à l'écrit en grande section. Thèse de doctorat en sociologie. Marseille : Université d'Aix-Marseille.

RICHARD-BOSSEZ, A. (2016). « La fiche à l'école maternelle : un objet littératié paradoxal ».

Recherches en éducation 25, p. 46-56.

\section{NOTES}

1. Nous ne traiterons dans cet article que de la présentation orale des savoirs par les enseignants, le rôle des fiches pédagogiques dans cette présentation a été traité par ailleurs (Richard-Bossez, 2016).

2. Nous utilisons le terme d'enseignant au masculin lorsque nous nous y référons de manière générique.

3. Les interactions enseignants-élèves ne sont pas les seules à se dérouler au cours des activités scolaires. De nombreuses interactions ont également lieu entre élèves ou entre les élèves et d'autres adultes présents dans les classes (ATSEM, AVS, enseignant spécialisé....). Nous ne développerons pas ici ces autres types d'interactions dont les effets sur les apprentissages sont plus aléatoires que celles qui se déroulent avec l'enseignant.

4. Par révision, on entend la possibilité pour un élève de "regarder à nouveau » les connaissances sur lesquelles il s'appuie quand un élément de la situation l'amène à les remettre en question. Ce processus de révision est donc celui qui permet de reprendre et reconfigurer ses savoirs initiaux quand ils ne sont pas adaptés à la situation proposée.

\section{RÉSUMÉS}

Dans cet article sont interrogées les activités relatives à l'écrit en maternelle au cours desquelles circulent des opérations relevant à la fois de l'oralité et de la littératie ainsi que la manière dont ces opérations s'articulent, ou non, dans les apprentissages des élèves. Le propos est illustré à 
partir de données issues d'une centaine d'heures d'observations réalisées lors d'une enquête de terrain de type ethnographique entre décembre 2010 et juin 2011 dans six classes de grande section situées dans des contextes sociaux variés (trois classes en « éducation prioritaire ", trois classes « ordinaires ») de la région toulonnaise. Trois dimensions du processus pédagogique sont développées : la présentation des savoirs relatifs à l'écrit par les enseignants; l'appropriation cognitive de ces savoirs par les élèves; les interactions entre enseignants et élèves, notamment pour ceux qui n'arrivent pas à saisir les savoirs en jeu sur le registre littératié attendu par l'école. Ce faisant, les différenciations à l'œuvre dans l'apprentissage de l'écrit sont questionnées.

This paper examines literacy learning activities in kindergarten where both oral and literacy related operations are carried out and how these operations are articulated, or not, in the learning process. The purpose is illustrated by data from about 100 hours of observations made during an ethnographic field survey between December 2010 and June 2011 in six kindergarten classes located in various social contexts (three "priority education" classes, three "ordinary" classes) in the South-East of France. Three dimensions of the pedagogical process are developed: the presentation of literacy knowledge by teachers; the cognitive assimilation of this knowledge by students; interactions between teachers and students, especially for those who have difficulties in acquiring knowledge on the literacy register expected by the school system. In doing so, this paper questions the school inequalities that can occur in literacy learning.

\section{INDEX}

Keywords : kindergarten, teaching-learning literacy, orality, literacy, school inequalities.

Mots-clés : école maternelle, enseignement-apprentissage de l'écrit, oralité, littératie, inégalités scolaires.

\section{AUTEUR}

\section{ARIANE RICHARD-BOSSEZ}

Aix-Marseille Université, CNRS, Lames, F-13007 Marseille, France 